\title{
SOLAR FLARE X-RAY MEASUREMENTS AND THEIR RELATION TO MICROWAVE BURSTS
}

\author{
L. D. DE FEITER
}

The Astronomical Institute, Utrecht, The Netherlands

\begin{abstract}
This review discusses the available observational material of solar hard X-ray bursts, their interpretation in terms of a model of the source region and their relation with other flash-phase phenomena, in particular the impulsive microwave bursts.
\end{abstract}

\section{Introduction}

This paper deals primarily with the flare-associated hard X-ray bursts and their relations to other simultaneously occurring flare phenomena, in particular the impulsive radio burst observed at microwaves and the metric type III bursts. Several examples can be found in the literature (e.g. Kane and Anderson, 1970; De Feiter, 1974). In terms of the solar sources of these radiations we will be dealing with high-energy electrons, in the $10-100 \mathrm{keV}$ range, originating during the flash phase of the flare, and their interactions with the ambient magneto-active plasma.

One of the important problems in this part of solar-flare research has already been stated fifteen years ago by Peterson and Winckler (1959) in their discussion of the event of 1958, March 20, the first recorded observation of a solar X-ray burst at photon energies above $10 \mathrm{keV}$. High-energy electrons can produce the X-ray burst through Coulomb-bremsstrahlung and the microwave burst through magneto-bremsstrahlung, but the numbers of electrons involved in the two mechanisms differ by a factor of $10^{4}$. Nevertheless the clear correlation in time of the two emissions points at a common source region, if not of the emissions themselves then of the electrons producing them.

A recent study by Kane (1972) has provided observational evidence that also the electrons that produce the metric type III bursts when moving outwards through the corona, are accelerated at the same time as those that give rise to the hard X-ray and the microwave bursts. Also, some impulsive EUV-bursts (e.g. Noyes, 1973; Donnelly, 1973) can be explained as enhanced emission of a region in the chromosphere-corona transition layer that is heated by penetrating electrons in the same energy range. When the emission mechanisms of all these emissions are properly understood observations in the various frequency domains can be used to determine more decisively the properties of the accelerated electrons, and the conditions under which the acceleration processes take place.

\section{Hard X-Ray Bursts}

(a) INSTRUMENTATION

Table I gives a summary of some of the characteristics of those satellite-born hard 
X-ray spectrometers for which the results are most extensively quoted in the literature. For a good comparison of the instrumental performances important parameters, like instrumental background, dynamic range, measures taken against pulse pile-up, efficiency curves, spectral resolution, procedures applied for the correction for instrumental effects etc., are needed. As the reports on these matters often are not always published, I have refrained from adding this information to the table. It should be noted though, that some of the older data do have shortcomings in this respect (e.g. Kane and Hudson, 1970). In terms of the effective collecting area and time resolution

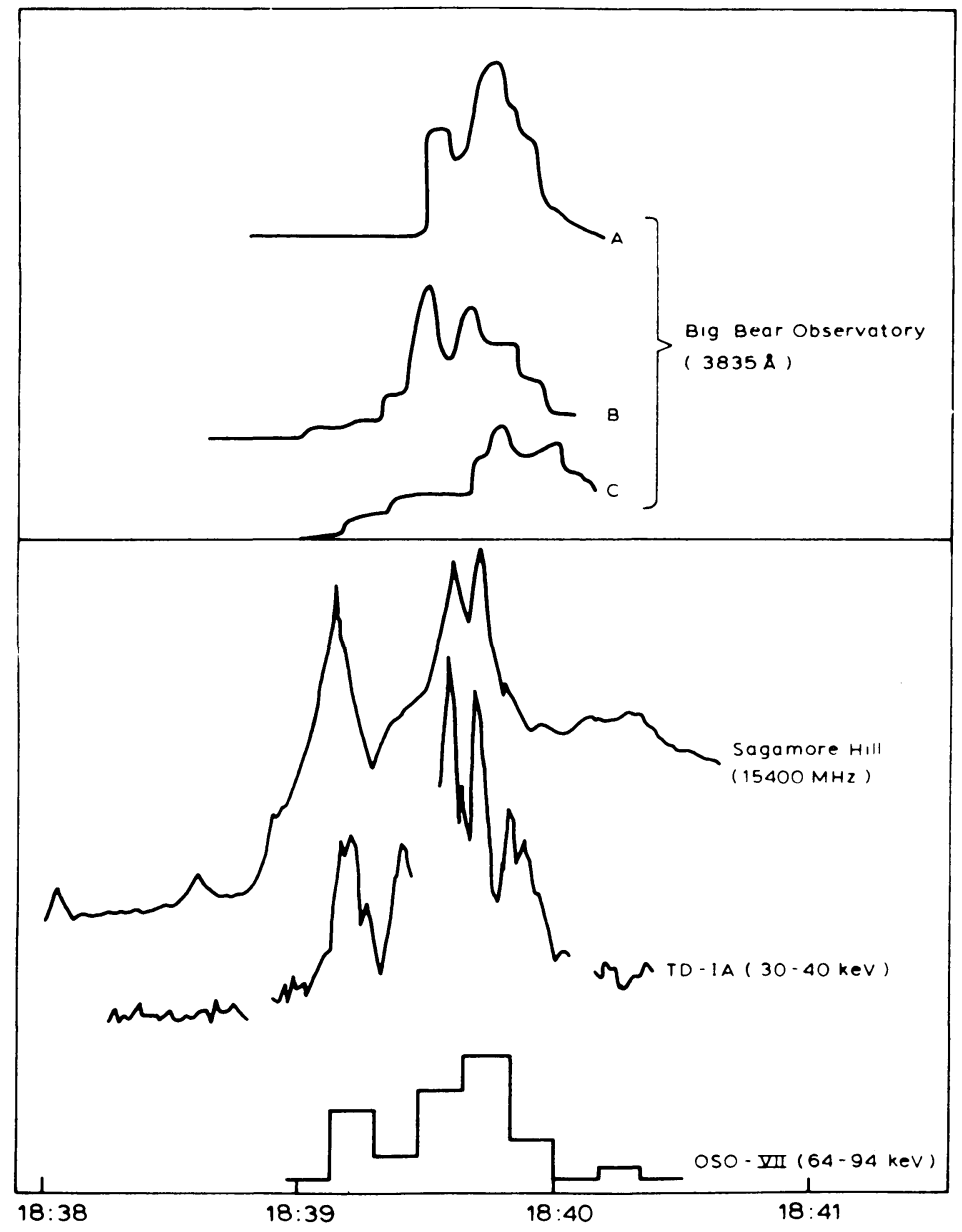

Fig. 1. Time profiles for a small flare of 1972, Aug. 2 at 1839 UT. From bottom to top the following profiles have been drawn: OSO 7 University of California, San Diego hard X-ray data (64-94 keV) taken at sampling intervals of $10 \mathrm{~s}$; ESRO TD 1A Space Research Laboratory Utrecht hard X-ray data (30-40 keV) taken at sampling intervals of $1.2 \mathrm{~s}$; Sagamore Hill microwave data $(15400 \mathrm{MHz})$ and intensity profiles of specific bright spots identified in Figure 10 of Zirin and Tanaka (1973), observed with the $15 \AA$ filter, centered on $\lambda .3835 \AA$. The value of high-time resolution hard X-ray data is clearly brought out in this figure. 


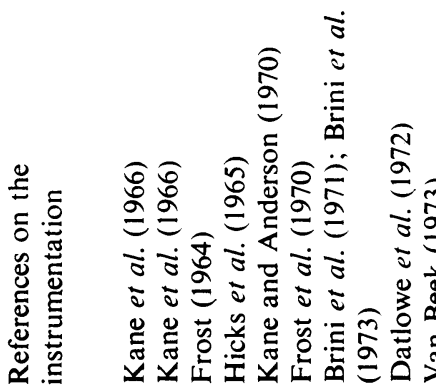

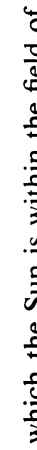

$\frac{\dot{0}}{\dot{0}}$

00

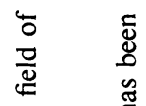

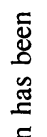

‡

I IIำ

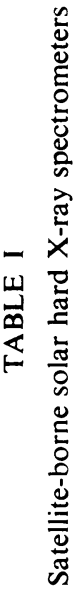

=

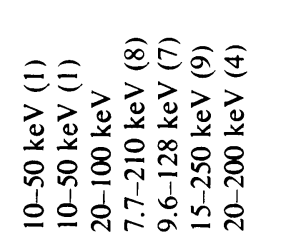

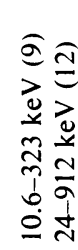

.

气 $\stackrel{\bigotimes}{\Xi}$

$\stackrel{\Xi}{\leftrightarrows}$ 
the experiments on board OGO 5, OSO 5 and TD 1A are about equal; the spectrometers on OSO 7 observe simultaneously the soft and the hard X-ray spectra which provides a possibility to separate clearly the thermal and the non-thermal components of solar X-ray bursts. Because of the highly eccentric orbit, the OGO 5 experiment operated for long stretches of time in a low-background environment outside the magnetosphere and therefore the data of this experiment are most suited for correlation studies with other flare phenomena; its dynamic range however is rather limited and restricts the observations to small flares. The importance of a resolution of time $1 \mathrm{~s}$ or less is clearly indicated in Figure 1 (see also Van Beek et al., 1974).

\section{(b) THE OCCURRENCE OF HARD X-RAY BURSTS}

Kane (1969) was able to show that many hard X-ray bursts, in the energy range around $20 \mathrm{keV}$, have a two-component structure, of which the first impulsive component has a hard non-thermal spectrum, whereas the second component varies slowly in time and has a soft thermal spectrum. The second component is absent above about $50 \mathrm{keV}$. Frost and Dennis (1971) discussed an event consisting of two hard non-thermal components. Peterson et al. (1973) find evidence for the existence of a slow hard X-ray phase usually associated with type IV radio emission. The relation between these three types of bursts is not completely clear at this moment; more information on this subject will be presented during this symposium. Here we will focus our attention primarily to the impulsive component. According to Kane and Anderson (1970) the impulsive component is essentially a flash-phase phenomena, and most flares with a well-pronounced flash-phase emit impulsive hard X-ray bursts.

\section{(c) TIME VARIATIONS}

When studied on a time scale of 1-2 s many hard X-ray bursts exhibit a clear fine structure. An example of this is given in Figure 1. Several other observations of rapid time variations have been reported; Beigman et al. (1971) have given indications of time scales as short as $0.05 \mathrm{~s}$ at wavelengths around $2 \AA$, Parks and Winckler (1971) and Frost (1969) also found rapid time variations. The high-time resolution data from the TD 1A experiment indicate that in some cases structures with characteristic times below $1 \mathrm{~s}$ do exist (Van Beek et al., 1974). Of particular interest in this connection is the event displayed in Figure 1. The rapid flashes found for this event are related to the fast and small flashes observed at $\lambda 3835$ by Zirin and Tanaka (1973) (see their Figure 10). The sizes of the sources of the individual UV flashes are as small as $1^{\prime \prime}$ in diameter. A preliminary comparison with the radio spectrograph data from the Harvard Radio Astronomy Station, Fort Davis (Maxwell, 1973) indicates that the X-ray peaks at 183930,183935 and 183943 may be associated with individual members of a group of type III bursts. Studies of this kind indicate strongly the necessity for a further reduction of the time resolution, also for ground-based observations. With modern data handling techniques it should be possible to overcome the problem of the tremendous data flow that will arise in that case. 


\section{(d) POLARIZATION}

Polarization of X-rays indicates an anisotropy in the velocity distribution of the highenergy electrons that produce the bremsstrahlung X-rays (e.g. Elwert and Haug, 1970). On several satellites in the Intercosmos series the group at the Lebedev Physical Institute in Moscow has flown Thomson scattering polarimeters (see e.g. Tindo et al. (1973) for the wavelength range $0.6-1.2 \AA$. As the polarization measurements will be discussed more fully in another paper during this Symposium it suffices to state here that in most cases polarization has been observed for a rather large fraction of the duration of the X-ray bursts concerned. This should be taken as an indication of a prolonged injection of directed beams of energetic electrons in these events. A small amount of polarization $(\sim 2 \%)$ can be attributed to Thomson scattering of part of the radiation by the photospheric layers.

\section{(e) POSITION AND STRUCTURE OF THE SOURCE REGION}

All observations discussed thusfar refer to total flux measurements for which the association with a particular flare is made on the basis of time coincidence. For hard $\mathrm{X}$-ray.bursts only one observation has been reported for which the position of the source region has been determined directly using a balloon-born collimator (Takakura et al., 1971). For this same event Tanaka and Enomé (1971) have determined the position line for the source of the associated microwave burst. The results of both measurements, drawn into a $\mathrm{H} \alpha$ filtergram of the optical flare, are presented in Figure 7 of Takakura et al., 1971; the intersection of the two position lines falls near a bright nucleus of the $\mathrm{H} \alpha$ flare. The size of the source was smaller than the spatial resolution of the instrument, i.e. I'. $^{\prime}$.

For the wavelength region between 1 and $10 \AA$ observations have been made with two crossed Soller-type collimators on board the cosmos 166 and 230, flown in 1967 (June 16-September 11) and 1968 (February 5-November 1) respectively (Beigman et al., 1969, 1971). The resolution of these collimators was about 15". The general structure, according to these observations of the X-ray flare is an elongated filament of $1^{\prime}-2$ ' length and about $15^{\prime \prime}$ width containing several small bright nuclei that may change rapidly in time.

The grazing incidence X-ray telescopes flown by the Solar Physics Group of A.S. \& E. on rockets (Vaiana et al., 1968; Vaiana and Giacconi, 1969) and on Skylab (Vaiana et al., 1973) operate in essentially the same wavelength region, i.e. 3.5-13.5. The spatial resolution of these instruments is better than $10^{\prime \prime}$ throughout the field of view; on some of the best exposures details of a few arcseconds can be resolved. The rocket observations of the flare of 1968 , June 8 , indicate the existence of a large looplike structure connecting two $\mathrm{H} \alpha$ emission centers on opposite sides of the magnetic neutral line.

The GSFC experiment on board OSO 7 contained among other experiments a multigrid collimator with a rectangular field of view of $20^{\prime \prime} \times 20^{\prime \prime}$ FWHM for the wavelength region 1.74-7.95 $\AA$. Observations of the flare of 1972, August 2 for which high-time resolution flash-phase phenomena are presented in Figure 1, confirm the 
earlier findings of an elongated shape with a length of about $27000 \mathrm{~km}\left(35^{\prime \prime}\right)$, but with a width that is smaller than the spatial resolution of the instrument.

Neither of the two last-mentioned reports refer to the bright points found by Beigman et al. (1969). However, comparison of hard X-ray time profiles with series of $\mathrm{H} \alpha$ filtergrams has led De Jager (1967) and Zirin and coworkers (Vorpahl and Zirin, 1970; Zirin et al., 1971; Vorpahl, 1972) to conclude that the $\mathrm{H} \alpha$ features that occur simultaneously with the hard X-ray burst are bright knots, often referred to as "flare kernels', with a diameter of $5^{\prime \prime}$ to $10^{\prime \prime}$ located near, to within $10^{\prime \prime}$, the magnetic neutral line (Vorpahl, 1972).

One observation that to some extent is in conflict with the results discussed thus far has been made during the very rare occasion of the occurrence of a solar flare in an active region that was being occulted by the Moon's limb during the eclipse of 1970 , March 7 (Kreplin and Taylor, 1971). The spatial resolution in this case was 20", determined by the time resolution and the velocity with which the Moon's limb moves over the solar disk. The total area of the region at $\lambda<16 \AA$ was found to be $136000 \mathrm{~km}$, or $3^{\prime}$, but it contained a hot dense core of $54000 \mathrm{~km}$ diameter, or 1'.2. These figures are larger than the ones quoted above by a factor of 5 to 10 . This discrepancy is probably due to the specific observing conditions, like e.g. the fact that the Moon's limb moved more or less along the flare filament. These observations seem to indicate that the hard X-ray emission takes place at the 'chromospheric footpoints' of a magnetic flux tube, whereas the hot and dense material along the whole flux tube emits $\mathrm{X}$-rays at longer wavelengths. In magnetically complex regions several of these flux tubes may brighten up rather independently, thus leading to short flashes in hard $\mathrm{X}$-rays at various places within the active region.

The relation between the hard and the soft X-ray bursts may be quite complex; in the initial phase the soft X-ray emission may indicate the presence of physical conditions that are favourable for the occurrence of impulsive phenomena and in the decay phase the soft $\mathrm{X}$-ray source contains a component that is produced through collisional heating by the non-thermal electrons required for the production of the hard $X$-ray burst. It seems to me that total flux measurements are of limited use in extending the analysis beyond a mere comparison of the energies involved in both emissions. A study of the evolution in time of specific regions of a flare, using high-resolution X-ray images in various spectral regions, are required in order to resolve these questions.

The photospheric magnetic field data discussed by Rust (1972) for the 2B flare of 1969, October 24 (see also Zirin et al., 1971) indicate the importance of small areas of one magnetic polarity embedded in a larger region of opposite polarity, the so-called 'satellite spots'. Important with respect to the occurrence of flares in these satellite spots is that they change with time; for this reason they were named 'Structures Magnétique Evolutives' by Martres et al. (1968).

(f) MODELS FOR THE HARD X-RAY EMISSION

The X-ray flux produced at the Earth's distance $R$ by energetic electrons with energy distribution $\mathrm{d} n_{\mathrm{c}}^{*} / \mathrm{d} E$ interacting in a region with ion density $n_{i}$, which may be in- 
homogeneously distributed through the volume $V$, is computed from

$$
\frac{\mathrm{d} F}{\mathrm{~d} E_{x}}=\frac{1}{4 \pi R^{2}} \int_{E_{x}}^{\infty} \mathrm{d} E_{x} / \int_{m}^{\mathrm{d} \sigma}\left(\int_{V} n_{\mathrm{i}} \frac{\mathrm{d} n_{\mathrm{e}}^{*}}{\mathrm{~d} E} \mathrm{~d} V\right) \mathrm{d} E .
$$

For the differential cross section the Bethe-Heitler formula can be used in most cases:

$$
\mathrm{d} E_{x}={ }_{\pi}^{2 \alpha \sigma_{0}} \frac{m c^{2}}{E E_{x}} \ln \left(\sqrt{E_{x}}+\sqrt{E_{x}}-1\right) .
$$

Isotropic emission has been assumed in (1); anisotropic emission due to relativistic beaming can be important at higher energies (e.g. Petrosian, 1973). The backscatter effects as studied by Tomblin (1972) and Santangelo et al. (1973) will not be discussed here; an interesting application of these effects which may allow a determination of the height above the photosphere of the emitting region will be reported during this symposium by Brown and Van Beek (1975).

Besides the spectrum of a hard X-ray burst its time evolution is an important observational datum; using the radio astronomical terminology we refer to the spectral development in time as the dynamic spectrum of the X-ray burst (Brown, 1971). This dynamic spectrum depends significantly upon the model of the source region, for which only a few limiting cases have been studied up till now. Brown (1972) analysed the so-called thin- and the thick-target models, where the differences are determined by the collisional losses of the energetic electrons, which are negligible in the former model and predominant in the latter. Also a distinction between models has been made on the bases of the time profile of the injection of energetic electrons into the source region; impulsive injection models (e.g. Takakura and Kai, 1966) and continuous injection models (e.g. Acton, 1968).

A comparison of these models should start with a discussion of the time-evolution equation of the non-thermal electron population (e.g. Blumenthal and Gould, 1970):

$$
\frac{\partial n_{\mathrm{e}}^{*}(E)}{\partial t}=\frac{\partial}{\partial E}\left(\frac{\mathrm{d} E}{\mathrm{~d} t} n_{\mathrm{e}}^{*}(E)\right)+q(E)-\frac{n_{\mathrm{e}}^{*}(E)}{\tau_{\mathrm{e}}}
$$

where the terms on the right-hand side indicate changes due to a systematic energy shift with time, e.g. collisional energy losses, the injection of energetic electrons from outside the emitting region and the escape from the emitting region respectively. If we indicate the characteristic times of these three processes by $\tau_{\mathrm{c}}, \tau_{\mathrm{a}}$ and $\tau_{\mathrm{e}}$ we can make the classification given in Table II.

As neither of these three characteristic times can be determined directly from the dynamic spectra the distinction between the four models listed in Table II cannot be made on the basis of the hard X-ray observations alone. However, assuming that the energy loss of the energetic electrons is entirely due to electron-electron collisions, a comparison of the observed decay times with the characteristic collisional loss time $\tau_{\mathrm{c}}$, 
and the variation of the decay time with energy allows a distinction to be made between the impulsive and continuous injection in the collision-dominated case. Energy considerations, and correlations with other flare phenomena provide means for a further discrimination.

TABLE II

Classification of hard X-ray emission models

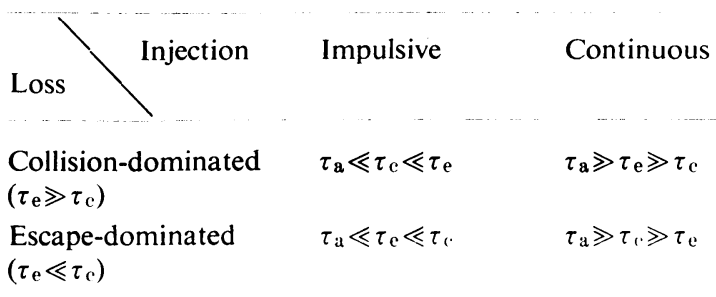

The energy loss rate through electron-electron collisions can be estimated from

$$
\mathrm{d} E=\frac{2 \pi r_{\mathrm{e}}^{2} m c^{4}}{v} n \ln \Lambda,
$$

where $r_{\mathrm{e}}$ is the classical electron radius and $\ln \Lambda$ is the Coulomb logarithm (cf. Petrosian, 1973 and references therein). The collisional decay time derived from (4) is

$$
\tau_{\mathrm{c}}=\frac{E^{3 / 2}}{\sqrt{2} 2 \pi r_{\mathrm{e}}^{2} m^{3 / 2} c^{4} n \ln \Lambda}=3.3 \times 10^{8} E_{n}^{3 / 2} \mathrm{~s},
$$

where in the numerical expression $E$ is in $\mathrm{keV}$.

If the observed decay times do not follow the $E^{3 / 2}$ behaviour, which is usually the case, we can conclude that the X-ray production takes place at high densities, or that the decay is determined by escape. In both cases a continuous injection of energetic electrons is required; in the former case, however, the total energy involved in the production of a given X-ray flux is smaller than in the second case.

Brown (1971), and several authors following him, has elaborated the thick-target case, which differs from the collision-dominated case introduced above. In that case the slowing down of the electrons is considered to happen in a time that is short as compared to the accumulation time of the instrument used for measuring the X-ray spectrum. Then we can follow, as Brown did, the X-ray production of a single electron until its energy is below the photon energy considered. Assuming $q(E)$ electrons with energy $E$ are injected per unit of time, the X-ray flux produced at the Earth's distance is given by

$$
\mathrm{d} F=\frac{1}{\mathrm{~d} E_{x}}=\frac{2 \alpha \sigma_{0}}{4 \pi R^{2}} \int_{\pi^{2} r_{\mathrm{e}}^{2} m c^{2} \ln \Lambda}^{\infty} Q(E) \ln \left(\sqrt{E_{x}}+\sqrt{E_{x}}+\sqrt{E_{x}}-1\right) \frac{\mathrm{d} E}{E_{x}},
$$

where $Q(E)=\int_{E}^{\infty} q(E) \mathrm{d} E$ is the integral injection spectrum. Equation (6) can also be derived from (1), using the appropriate solution of (3) for the instantaneous energy spectrum. The thick-target assumption means that there is at all times an equilibrium 
between the instantaneous energy spectrum of the energetic electron population and the spectrum of the injected electrons, i.e.

$$
\frac{\mathrm{d}}{\mathrm{d} E}\left(-\frac{\mathrm{d} E}{\mathrm{~d} t} \frac{\mathrm{d} n_{\mathrm{e}}^{*}(E)}{\mathrm{d} E}\right)=q(E),
$$

of which the solution is

$$
\frac{\mathrm{d} n_{\mathrm{e}}^{*}(E)}{\mathrm{d} E}=\frac{Q(E)}{-\mathrm{d} E / \mathrm{d} t} .
$$

Substitution of (8) and (4) into (1) then leads to (6).

Similarly we can define the thin-target case, i.e. by requiring that there exists an equilibrium between the injected and the escaping electrons. In that case we have

$$
\frac{\mathrm{d} n_{\mathrm{e}}^{*}(E)}{\mathrm{d} E}=q(E) \tau_{\mathrm{e}} .
$$

Because of the lack of information on $\tau_{\mathrm{e}}$, in particular its energy dependence, the conclusion for the injection spectrum $q(E)$ derived from the observations of hard $\mathrm{X}$-ray spectra are not as certain as in the thick-target case. The photon flux for the thin-target model then becomes

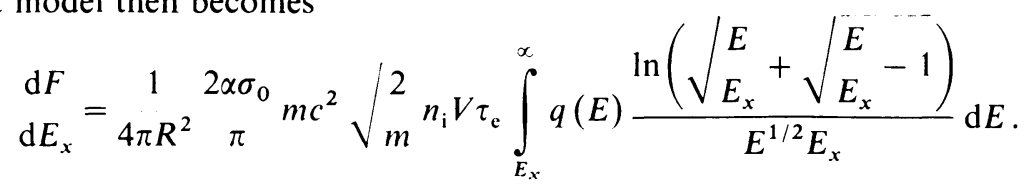

For a power-law injection spectrum, valid above a certain cut-off energy $E_{\mathrm{c}}$, for which the total kinetic energy contents is $P_{\mathrm{K}} \mathrm{keV} \mathrm{s}^{-1}$ and the spectral index is $\delta$, we find for the $\mathrm{X}$-ray fluxes for the thick- and the thin-target case respectively:

and

$$
\begin{aligned}
\left(\begin{array}{c}
\mathrm{d} F \\
\mathrm{~d} E_{x}
\end{array}\right)_{\text {thick }}= & 3.46 \times 10^{-34} \frac{B\left(\delta-2, \frac{1}{2}\right)}{2(\delta-1)} \times \\
& \times E_{\mathrm{c}}^{-1}\left(E_{x} / E_{\mathrm{c}}\right)^{-\delta+1} P_{\mathrm{K}} \mathrm{cm}^{-2} \mathrm{~s}^{-1} \mathrm{keV}^{-1}
\end{aligned}
$$

$$
\begin{aligned}
\left(\begin{array}{l}
\mathrm{d} F \\
\mathrm{~d} E_{x}
\end{array}\right)_{\text {thin }}= & 1.05 \times 10^{-42} \frac{(\delta-2) B\left(\delta-\frac{1}{2}, \frac{1}{2}\right)}{2(\delta-1)} \times \\
& \times n_{\mathrm{i}} \tau_{\mathrm{e}} E_{\mathrm{c}}^{-5 / 2}\left(E_{x} / E_{\mathrm{c}}\right)^{-\delta-1 / 2} P_{\mathrm{K}} \mathrm{cm}^{-2} \mathrm{~s}^{-1} \mathrm{keV}^{-1}
\end{aligned}
$$

In these equations $B(x, y)$ is the Beta function (see also Brown, 1971) and all energies are expressed in $\mathrm{keV}$. In order to produce a hard $\mathrm{X}$-ray burst of the same size the ratio $R$ of the kinetic energy of the energetic electrons required in the two limiting cases is

$$
\begin{aligned}
R=\frac{P_{\mathrm{K}, \text { thick }}}{P_{\mathrm{K}, \text { thin }}}= & 3.03 \times 10^{-9} \frac{2(\delta-1)(\delta-2) B\left(\delta-\frac{1}{2}, \frac{1}{2}\right)}{(2 \delta-1) B\left(\delta-2, \frac{1}{2}\right)} \times \\
& \times n_{\mathrm{i}} \tau_{\mathrm{e}} E_{\mathrm{c}}^{-3 / 2}\left(E_{x} / E_{\mathrm{c}}\right)^{-3 / 2}
\end{aligned}
$$


Without further specification of $n_{i}$ and $\tau_{\mathrm{e}}$ this ratio cannot be determined. From future observations of the structures of the hard and soft X-ray emitting regions we may find some indications as to these physical parameters; for the time being we can only give estimates. As $\tau_{\mathrm{e}}$ should certainly be smaller than $\tau_{\mathrm{c}}$ (Equation (5)), we find that in all practical cases $R<1$, which means that the thick-target case is more efficient with respect to X-ray production than the thin-target case, which of course is more or less obvious.

With respect to the energy and mass balance for the flare flash phase phenomena, the thick-target case should be studied with preference, not only because of the fact that its analysis is rather straightforward, but especially because it requires the least amount of energy and mass in order to produce an X-ray burst of the observed size and thus it gives lower limits for these two important quantities. Converting (11) to an energy flux instead of a photon flux we find the following relation

$$
P_{\mathrm{K}, \text { thick }}=5.78 \times 10^{33} \begin{gathered}
\delta-1 \\
B\left(\delta-2, \frac{1}{2}\right)
\end{gathered}\left(E_{x} / E_{\mathrm{c}}\right)^{\delta-2} \frac{\mathrm{d} S_{x}}{\mathrm{~d} E_{x}} \mathrm{keV} \mathrm{s}^{-1},
$$

where $\mathrm{d} S_{x} / \mathrm{d} E_{x}$ is expressed in $\mathrm{keV} \mathrm{cm}^{-2} \mathrm{~s}^{-1} \mathrm{keV}^{-1}$. The energies involved in the energetic electrons range from $10^{28} \mathrm{erg}$, in small spikes, to $10^{30}-10^{31} \mathrm{erg}$ and more, in long-enduring bursts as e.g. the one of 1972, August 4.

\section{Microwave Bursts}

\section{(a) TYPES OF MICROWAVE BURSTS}

The radio bursts as observed at microwave frequencies, i.e. above $1000 \mathrm{MHz}$, can be classified into three main categories: gradual bursts, impulsive bursts and type IV bursts (Boischot, 1972). In some cases the microwave burst can be decomposed into a gradual and an impulsive burst; the gradual burst may start before the impulsive burst (precursor) and it may attain its maximum after the impulsive burst (post-burst increase). The gradual bursts are generally fairly weak and they show a clear resemblance to the soft X-ray bursts. Following the original suggestion of Kawabata (1960) they are interpieted as due to thermal bremsstrahlung; recent analyses of Hudson and Ohki (1972) and of Shimabukuro (1972) have confirmed the common origin of the gradual microwave and the soft $\mathrm{X}$-ray burst in a region with emission measure between $10^{49}$ and $10^{50} \mathrm{~cm}^{-3}$ and temperatures in the range of $2-6 \times 10^{6} \mathrm{~K}$. According to Ramaty and Petrosian (1972) a thermal interpretation is only possible for the lowintensity gradual microwave bursts; the impulsive microwave as well as the microwave type IV bursts have a non-thermal nature. Of these non-thermal bursts the impulsive ones are closely related to the hard X-ray bursts.

The intensities of impulsive microwave bursts vary within wide limits, ranging from about $40 \mathrm{sfu}\left(1 \mathrm{sfu}=10^{-22}\right.$ watt $\left.\mathrm{m}^{-2} \mathrm{~Hz}^{-1}\right)$ to more than $10^{4} \mathrm{sfu}$; the largest burst ever recorded at microwavelengths reached a flux level of $47000 \mathrm{sfu}$ at $71 \mathrm{GHz}$ (Croom, 1970). All strong microwave bursts $(\gtrsim 100 \mathrm{sfu})$ are associated with flares. 
whereas for weak bursts ( $\lesssim 40 \mathrm{sfu}$ ) the correlation is much weaker, about $65 \%$ (Kundu, 1965). This correlation in general is better for bursts at millimeter wavelengths (Shimabukuro, 1968; Croom and Powell, 1971). The association of microwave bursts and flares is different for different active regions; in active regions with magnetically complex configurations almost all flares are accompanied by microwave bursts (Fokker and Roosen, 1961; Matsuura and Nave, 1971).

\section{(b) SPECTRAL CHARACTERISTICS}

The spectrum of a microwave burst is a broad-band continuum with a maximum intensity around 3-10 GHz (Takakura, 1967). The frequency at which the maximum intensity occurs shifts to progressingly higher values with increasing intensity, also the average intensity of the bursts with the same $f_{\max }$ is proportional to $f_{\max }^{2}$ (Fürst, 1971). Hagen and Neidig (1971) have found a distinct difference in spectral characteristics for bursts that are associated with flares that occur very near or over sunspots and those that occur away from sunspots; the former have heir maximum at frequencies above $10 \mathrm{GHz}$, whereas for the latter type of bursts the maximum emission occurs near 3.GHz. Also Guidice and Castelli (1973) noted a tendency for the higher values of $f_{\max }$ to occur in active regions in which the spots have a large magnetic field strength. These same authors found a linear relation between the low-frequency cut-off, $f_{\text {cut }}$ and $f_{\max }$, which for medium-size events (50-500 sfu) can be represented by $f_{\max }=3.4 f_{\text {cut }}$.

The relation between certain spectral characteristics of microwave bursts to solar proton acceleration has recently been discussed by Croom (1973, and references therein), who found that the association rate of microwave bursts and proton events increases with $f_{\max }$ and that the proton flux is related to the duration of the burst. Guidice and Castelli (1973) mention the necessity for the radio wave burst to have both strong microwave and meterwave components for it to be associated with a proton flare. These results are essentially an extension of earlier findings by e.g. Kundu and Haddock (1960) and others (see discussion by Kundu, 1965). To the present reviewer it seems that it is the microwave type IV burst rather than the impulsive microwave burst that is meant in these studies.

\section{(c) POSITION AND STRUCTURE OF THE SOURCE REGION}

The position of the source of the microwave burst corresponds roughly with that of the optical flare (Kundu, 1965), when determined with an interferometer having a resolution of a few arcminutes. Usually the source is more or less stationary, but in some cases a displacement can be observed. An interesting event in this connection is the flare of 1969, March 30 (Enomé and Tanaka, 1971), which was also exceptional in other respects (see e.g. Frost and Dennis, 1971; Smerd, 1970). The flare occurred $10^{\circ}$ behind the west limb of the Sun, which means that the burst source was at $10000 \mathrm{~km}$ or higher. After the burst maximum the source expanded to reach an altitude of $200000 \mathrm{~km}\left(0.3 R_{\odot}\right)$. Enomé and Tanaka interpret this expansion in terms of diffusion of the energetic electrons in a turbulent magnetic field. It should be noted, however, that in between the two components of the burst a type II radio burst was 
observed. This prompted Frost and Dennis (1971) to suggest that the second component was due to electrons accelerated by repeated reflections at the shock front that produced the type II radio burst.

Recent observations with a 24 " fan beam show that the angular size of the source of the impulsive burst is about 0.5 or less (Enomé et al., 1969), at $3.75 \mathrm{GHz}$. Also several cases of multicomponent bursts have been observed, which show independent intensity variations; to within the accuracy of the observations the sources of these components coincide with individual sunspots (Enomé and Tanaka, 1973). All these observations indicate that the microwave burst is produced near the feet of magneticfield loops; in some cases emission takes place at both ends of such a loop with opposite senses of polarization.

\section{(d) MODELS FOR HARD X-RAY AND MICROWAVE EMISSION}

The theory of the radio emission and absorption of non-, or near relativistic electrons in a magnetic field has been elaborated by many authors, notably Ginzburg and Syrovatskii (1965, 1969), Takakura (1967), Ramaty (1969, 1973). In particular the emission- and absorption characteristics in a magneto-active plasma are still being debated (e.g. Ko, 1973). However, the theory may still not be in its final shape, the models to which the theory thus far has been applied are at best zero order approximations of the situation likely to exist in the solar atmosphere (e.g. Takakura and Kai, 1966; Holt and Ramaty, 1969; Takakura, 1972). The combination of these two circumstances makes the whole problem rather intractable, in particular when applied to total flux measurements in a frequency range of which a significant part corresponds to the plasma frequencies to be expected in the source region.

Briefly the problem can be stated as follows. The energy loss due to magnetic bremsstrahlung of a non-relativistic electron in a magnetic field with field strength $B$ is

$$
\left(\begin{array}{l}
\mathrm{d} E \\
\mathrm{~d} t
\end{array}\right)_{\mathrm{mb}}=-3.89 \times 10^{-9} B^{2} E,
$$

where $B$ is in Gauss. This energy is, depending on $E / m c^{2}$, emitted mainly in the frequencies near the local cyclotron frequency $f_{\mathrm{H}} \approx 2.8 \times 10^{6} \mathrm{~B} \mathrm{~Hz}$, and a few low harmonics of this frequency. The total energy emitted by an ensemble of energetic electrons, $E>E_{\mathrm{c}}$, with total kinetic energy $P_{\mathrm{K}}$, then is

$$
P_{\mathrm{mb}}=3.89 \times 10^{-9} B^{2} P_{\mathrm{K}} \text {. }
$$

The radio flux at the Earth's distance, assuming isotropic emission, is

$$
S_{v}=1.38 \times 10^{-39} B^{2} P_{\mathrm{K}} \mathrm{sf}^{-1} \text { watt } \mathrm{m}^{-2} \mathrm{~Hz}^{-1} \text {. }
$$

For the maximum of the burst of 1966, July 7 we find from Takakura (1972): $S_{v, \max } \approx 10^{-18}$ watt $\mathrm{m}^{-2} \mathrm{~Hz}^{-1}, f_{\max } \approx 10^{10} \mathrm{~Hz}, \Delta f \approx 2 \times 10^{10} \mathrm{~Hz}$. Hence the kinetic energy $P_{\mathrm{K}}$ of the electrons required to produce this radio flux is $P_{\mathrm{K} \text {, radio }} \approx 1.44 \times 10^{31} B^{-2}$ erg s $^{-1}$. The hard X-ray photon spectrum can be represented by $\mathrm{d} F / \mathrm{d} E_{x}=6\left(E_{x} / 100\right)^{-\gamma}$ photons $\mathrm{cm}^{-2} \mathrm{~s}^{-1} \mathrm{keV}^{-1}$, where $\gamma$ is about 3. Using (11), we find for the kinetic 
energy $P_{\mathrm{K}, x}$ of the electrons required to produce this X-ray flux $2 \times 10^{28} \mathrm{erg} \mathrm{s}^{-1}$ for $E_{\mathrm{c}}=100 \mathrm{keV}$. If we take $f_{\max } \simeq 3 f_{\mathrm{H}}$, the magnetic field strength in the source region should be: $B \approx 1200 \mathrm{G}$ and therefore $P_{\mathrm{K} \text {, radio }} \approx 10^{25} \mathrm{erg} \mathrm{s}^{-1}$. Hence the kinetic energy of the electrons that produce the hard $\mathrm{X}$-ray burst is $10^{3}-10^{4}$ times that of the electrons that emit the microwave burst. This discrepancy, already noted by Peterson and Winckler (1959), has been extensively discussed over the past fifteen years; essential in all these discussions is the reduction of the radio emission of an energetic particle population due to the plasma effects; self absorption by the energetic electrons themselves, reduction of emissivity, absorption by the layers overlying the source region, non-isotropic pitch angle distribution and so on. These problems will be discussed during this symposium by Takakura (1975) and therefore I will not go into details of the computation of the radio spectrum. It suffices to state here that Takakura (1972) succeeded to make the two figures approach each other to within the uncertainties of the measurements, and of the assumptions of the model. It seems to me that this is about the maximum we can hope to obtain from total flux measurements. Real progress from here onwards seems to be possible only on the basis of spatially resolved observations for both the microwave and the hard X-ray region.

\section{References}

Acton, L. W.: 1968, Astrophys. J. 152, 305.

Beigman, I. L., Grineva, Yu. I., Mandel'shtam, S. L., Vainshtein, L. A., and Zhitnik, I. A.: 1969, Solar Phys. 9, 160.

Beigman, I. L., Vainshtein, L. A., Vasilyev, B. H., Zhitnik, B. N., Ivanov, V. D., Korneyev, V. V., Krutov, V. V., Mandel'shtam, S. L., Tindo, I. P., and Shurygin, A. I.: 1971, Kosmich. Isled. 9, 123.

Blumenthal, G. R. and Gould, R. J.: 1970, Rev. Mod. Phys. 42, 237.

Boischot, A.: 1972, in E. R. Dyer (General Ed.), Solar-Terrestrial Physics, Part I, D. Reidel Publ. Co., Dordrecht, Holland, p. 87.

Brini, D., Evangelisti, F., Fuligni Di Grande, M. T., Pizzichini, G., Spizzichino, A., and Vespigani, G. R.: 1973, Astron. Astrophys. 25, 17.

Brini, D., Fuligni, F., Evangelisti, F., and Vespigani, G.: 1971, Nuovo Cimento, Series 11, 28.

Brown, J. C.: 1971, Solar Phys. 18, 489.

Brown, J. C.: 1972, Solar Phys. 25, 158.

Brown, J. C. and Van Beek, H. F.: 1975, This volume, p. 239.

Croom, D. L.: 1970, Solar Phys. 15, 414.

Croom, D. L.: 1973, in R. Ramaty and R. G. Stone (eds.), High-Energy Phenomena on the Sun, NASA SP-342 p. 114.

Croom, D. L. and Powell, R. J.: 1971, Solar Phys. 20, 136.

Datlowe, D. W., McKenzie, D. L. and Peterson, L. E. : 1972, Univ. California, San Diego SP-72-06.

De Feiter, L. D.: 1974, Space Sci. Rev. 16, 3.

De Jager, C.: 1967, Solar Phys. 2, 327.

Donnelly, R. F.: 1973, in R. Ramaty and R. G. Stone (eds.), High-Energy Phenomena on the Sun, NASA SP-342, p. 242.

Elwert, G. and Haug, E.: 1970, Solar Phys. 15, 234.

Enomé, S. and Tanaka, H.: 1971, in R. Howard (ed.), 'Solar Magnetic Fields', IAU Symp. 43, 413.

Enomé, S. and Tanaka, H.: 1973, in R. Ramaty and R. G. Stone (eds.), High-Energy Phenomena on the Sun, NASA SP-342, p. 78.

Enomé, S., Kakinuma, T., and Tanaka, H.: 1969, Solar Phys. 6, 428.

Fokker, A. D. and Roosen, J.: 1961, Bull. Astron. Inst. Neth. 16, 86.

Frost, K. J.: 1964, in W. N. Hess (ed.), The Physics of Solar Flares, NASA SP-50, p. 139 
Frost, K. J.: 1969, Astrophys. J. Letters 158, L159.

Frost, K. J. and Dennis, B. R.: 1971, Astrophys. J. 165, 655.

Frost, K. J., Dennis, B. R., and Lencho, R. J.: 1970, GSFC X-680-70-440.

Fürst, E.: 1971, Solar Phys. 18, 84.

Ginzburg, V. L. and Syrovatskii, S. I.: 1965, Ann. Rev. Astron. Astrophys. 3, 297.

Ginzburg, V. L. and Syrovatskii, S. I.: 1969, Ann. Rev. Astron. Astrophys. 7, 375.

Guidice, D. A. and Castelli, J. P.: 1973, in R. Ramaty and R. G. Stone (eds.), High-Energy Phenomena on the Sun, NASA SP-342, p. 87.

Hagen, J. P. and Neidig, D. F.: 1971, Solar Phys. 18, 305.

Hicks, D. B., Reid, L., and Peterson, L. E.: 1965, IEEE Trans. Nucl. Sci. 12, 54.

Holt, S. S. and Ramaty, R.: 1969, Solar Phys. 8, 119.

Hudson, H. S. and Ohki, K.: 1972, Solar Phys. 23, 155.

Kane, S. R.: 1969, Astrophys. J. Letters 157, L139.

Kane, S. R.: 1972, Solar Phys. $27,174$.

Kane, S. R. and Anderson, K. A.: 1970, Astrophys. J. 162, 1003.

Kane, S. R. and Hudson, H. S. : 1970, Solar Phys. 14, 414.

Kane, S. R., Pfitzer, K. A., and Winckler, J. R.: 1966, Univ. of Minnesota Techn. Rept. CR-87.

Kawabata, K.: 1960, Rept. Ion. Space Res. Japan 14, 405.

Ko, H. C.: 1973, in R. Ramaty and R. G. Stone (eds.), High-Energy Phenomena on the Sun, NASA SP-342, p. 198.

Kreplin, R. W. and Taylor, R. G.: 1971, Solar Phys. 21, 452.

Kundu, M. R.: 1965, Solar Radio Astronomy, Interscience Publishers, New York.

Kundu, M. R. and Haddock, F. T.: 1960, Nature 186, 610.

Martres, M.-J., Michard, R., Soru-Iscovici, I., and Tsap, T. T.: 1968, Solar Phys. 5, 187.

Matsuura, O. T. and Nave, M. F. F.: 1971, Solar Phys. 16, 417.

Maxwell, A.: 1973, in H. E. Coffey (ed.), Collected Data Reports on August 1972 Solar-Terrestrial Events, Report UAG-28 Part I, p. 255.

Neupert, W. M., Thomas, R. J., and Chapman, R. D.: 1974, Solar Phys. 34, 349.

Noyes, R. W.: 1973, in R. Ramaty and R. G. Stone (eds.), High-Energy Phenomena on the Sun, NASA SP-342, p. 231.

Parks, G. K. and Winckler, J. R.: 1971, Solar Phys. 16, 186.

Peterson, L. E. and Winckler, J. R.: 1959, J. Geophys. Res. 64, 697.

Peterson, L. E., Datlowe, D. W., and McKenzie, D. L.: 1973, in R. Ramaty and R. G. Stone (eds.), High-Energy Phenomena on the Sun, NASA SP-342, p. 132.

Petrosian, V.: 1973, Astrophys. J. 186, 291.

Ramaty, R.: 1969, Astrophys. J. 158, 753.

Ramaty, R.: 1973, in R. Ramaty and R. G. Stone (eds.), High-Energy Phenomena on the Sun, NASA SP-342, p. 188.

Ramaty, R. and Petrosian, V.: 1972, Astrophys. J. 178, 241.

Rust, D. M.: 1972, Solar Phys. 25, 141.

Santangelo, N., Horstman, H., and Horstman-Moretti, E.: 1973, Solar Phys. 29, 143.

Shimabukuro, F. I.: 1968, Solar Phys. 5, 498.

Shimabukuro, F. I.: 1972, Solar Phys. 23, 169.

Smerd, S. F.: 1970, Proc. Astron. Soc. Australia, 1, 305.

Takakura, T.: 1967, Solar Phys. 1, 304.

Takakura, T.: 1972, Solar Phys. 26, 151.

Takakura, T.: 1975, This volume, p. 299.

Takakura, T. and Kai, K.: 1966, Publ. Astron. Soc. Japan 18, 57.

Takakura, T., Ohki, K., Shibuya, N., Fujii, M., Matsuoka, M., Miyamoto, S., Nishimura, J., Oda, M., Ogawary, Y., and Ota, S.: 1971, Solar Phys. 16, 454.

Tanaka, H. and Enomé, S.: 1971, Solar Phys. 17, 408.

Tindo, I. P., Mandel'shtam, S. L., and Shuryghin, A. I.: 1973, Solar Phys. 32, 469.

Tomblin, F. F.: 1972, Astrophys. J. 171, 377.

Vaiana, G. S., Davis, J. M., Giacconi, R., Krieger, A., Silk, J. K.. Timothy, A. F., and Zombeck, M. : 1973, Astrophys. J. Letters, 185, L47.

Vaiana, G. S. and Giacconi, R.: 1969, in D. G. Wentzel and D. A. Tidman (eds.), Plasma Instabilities in Astrophysics, p. 91. 
Vaiana, G. S., Reidy, W. P., Zehnpfennig, T., Van Speybroeck, L., and Giacconi, R.: 1968, Science $161,564$.

Van Beek, H. F.: 1973, 'Development and Performance of a Solar Hard X-Ray Spectrometer', Utrecht University (Ph.D. Thesis).

Van Beek, H. F., De Feiter, L. D., and De Jager, C.: 1974, Space Res. 14, 447.

Vorpahl, J.: 1972, Solar Phys. 26, 397.

Vorpahl, J. and Zirin, H.: 1970, Solar Phys. 11, 285.

Zirin, H. and Tanaka, K.: 1973, Solar Phys. 32, 173.

Zirin, H., Pruss, G., and Vorpahl, J.: 1971, Solar Phys. 19, 463. 